\title{
Development of High-Efficiency Low-Cost Drive System of Small- Size Electric Vehicles
}

\author{
Thuy-Lien Duong*, Thanh-Vu Tran*, Tae-Won Chun*, Hong-Hee Lee*, and \\ Heung-Geun Kim*
}

\begin{abstract}
This paper designs the high-efficiency and the low-cost drive system of the smallsize electric vehicles (EVs). The power circuit for driving the de motor is designed by considering both the cost and efficiency. In order to reduce the conduction loss of MOTFET and diode for controlling an armature voltage, some MOSFETs and diodes at the armature are in parallel connection. An operating sequence for both the field current and the armature voltage according to the accelerator pedal angle is suggested for changing smoothly the rotating direction of dc motor. Through the simulation studies, the performances of the proposed methods are verified.
\end{abstract}

Keywords: Electric vehicle, conduction loss, buck converter, switching loss, DC motor

\section{Introduction}

Recently, because environmental pollution and the energy crisis are rising globally, electric vehicles (EVs) are promising instead of traditional gasoline vehicles. As the EVs are driven by electricity stored in a battery, they still suffer from the main problem as short driving range due to the low energy content of the batteries. As how to use the battery's energy efficiently is a hot issue for developing EVs, it is important to design the drive system of a traction motor with high efficiency.

Selection of traction motors for propulsion system is very important step that requires special attention. In fact, the choice of electric-propulsion systems for EVs mostly depends on three factors: efficiency, cost, and reliability. In an industrial point of view, the major types of electric motors adapted or under serious consideration for EVs include the dc motor, an induction motor (IM), and brushless DC (BLDC) motor. The BLDC motor has been widely used in the EVs, because it provides high power density, and has higher efficiency than induction motor [1]-[3]. By the way, in order to drive the BLDC motor, the 3-phase PWM inverter and complex control system are required [4]. Recently, the price of PM has been dramatically raised. They cause the increase of the manufacturing cost of the EVs.

The small-size EVs such as the golf car and scooters require the simple propulsion system in order to reduce the

\footnotetext{
* Dept.. of Electrical Engineering, Univ. of Ulsan, Korea. (dthuylien12@yahoo.com,tranthanhvu101@yahoo.com,twchun@mail. ulsan.ac.kr,hhlee@mail.ulsan.ac.kr,kimhg@knu.ac.kr)

Received 15 April 2012; Accepted 18 May 2012
}

manufacturing cost. Although a brushed de motor has some problems such as requirement of maintaining both the brush and commutation, it has some advantages like a simple drive system and high starting torque. Thus, the dc motor as the traction motor is more suitable at the low cost small-size EVs [5].

In this paper, the power circuit for driving the dc motors is designed by considering both the cost and efficiency. In order to reduce the conduction loss of MOTFET and diode for controlling an armature voltage, some MOSFETs and diodes at the armature are in parallel connection. An algorithm to change smoothly the rotating direction of the motor is suggested. In order to verify the performances of proposed methods, some simulation studies are carried out.

\section{Drive circuit of de motor}

The power circuit for driving the dc motors is designed by considering both the cost and efficiency. The rating of $\mathrm{dc}$ motor is as follow.

- Output power: $3.8[\mathrm{~kW}]$

- Armature voltage, $V_{a}: 48[\mathrm{~V}]$

- Armature current, $I_{a}: 100[\mathrm{~A}]$

- Field current, $I_{f}: 10[\mathrm{~A}]$

As the battery is used as the dc source, the dc input voltage is $48 \mathrm{~V}$, which is the battery voltage. Therefore, the rated armature current is very high in order to produce the required output power. The dc motor as the traction motor of the EVs must rotate at the forward/reverse directions. 
In order to satisfy this requirement, the general method is to change the polarity of armature voltage by using an $\mathrm{H}$ bridge type circuit as shown in Fig. 1. Although it is easy to change the rotating direction of motor by changing the polarity of the armature voltage, the four switching devices with high current capacity in the H-bridge circuit are required. It increases the cost of the drive circuit, and decreases the efficiency by large conduction losses of switching devices.

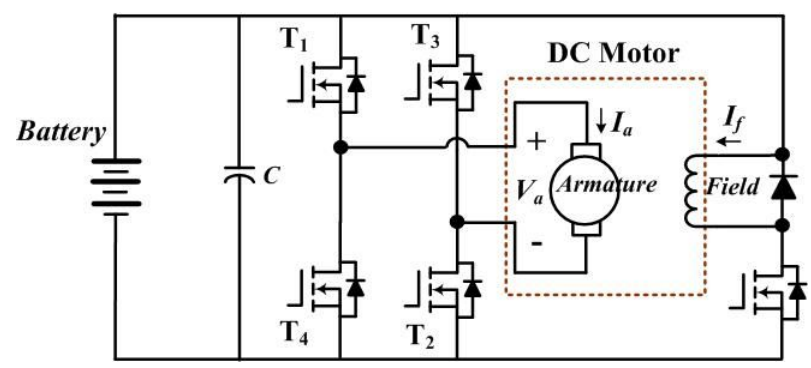

Fig. 1. Traditional drive circuit of de motor.

Fig. 2 shows the drive circuit used at the paper. The armature voltage is controlled by the buck converter consisting of one MOSFET and diode, and the H-bridge type circuit is used to control the field current and also change the flowing direction of the current for changing the forward/reverse directions of motor. As the current capacity of the four MOSFETs of the H-bridge circuit is much low, the cost of the drive circuit can be reduced.

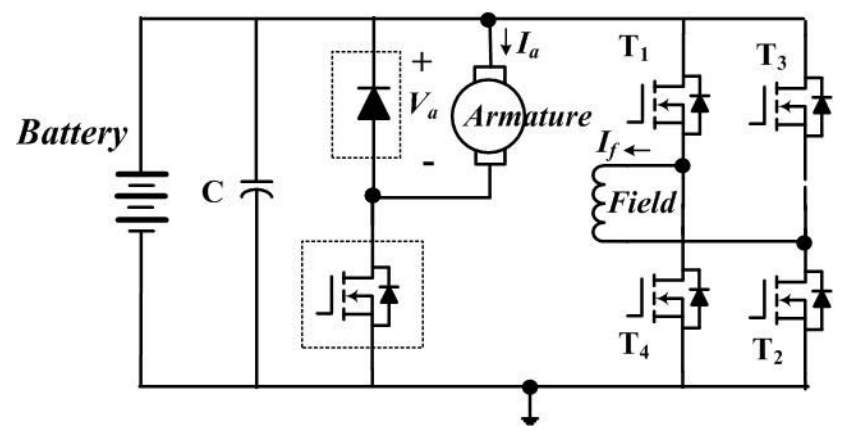

Fig. 2. Drive circuit of dc motor for traction.

\section{Reduction of losses of switching devices}

\subsection{Reduction of conduction loss of switching device}

In order to reduce the conduction losses of both MOSFET and diode at the buck converter for controlling an armature voltage, some MOSFETs and diodes are in the parallel connection. When the $n$ MOSFETs are connected in parallel, the equivalent resistance of all MOSFETs becomes $\left(r_{d s(o n)} / n\right)$, where $r_{d s(o n)}$ is an equivalent resistance between drain and source when MOSFET is in the conduction state. The total conduction loss of all MOSFETs of the buck converter $P_{C F}$ can be derived as [6],[7]

$$
P_{C F}=I_{a}^{2} \cdot\left(\frac{r_{d s(o n)}}{n}\right)
$$

,where $I_{a}$ is the armature current. The conduction loss is dependent on the number of MOSFETs connected in parallel.

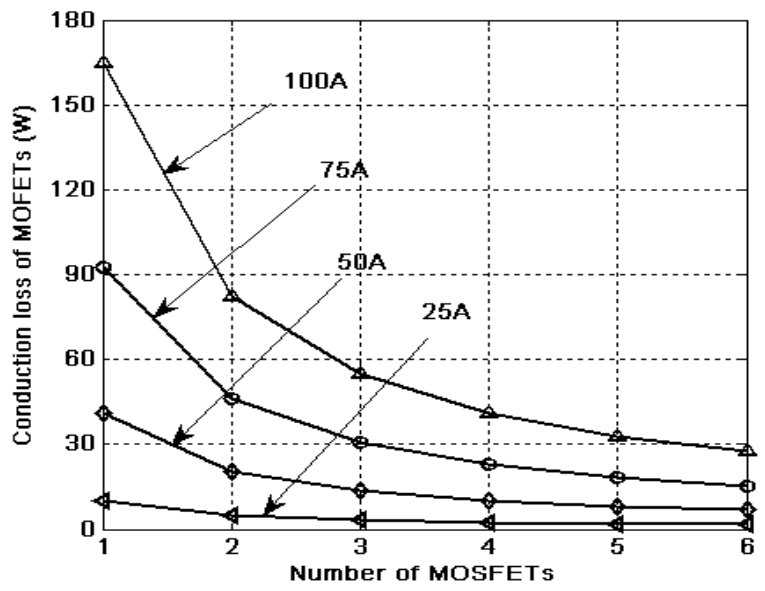

Fig. 3. Conduction loss with a variation of number of MOSFETs in parallel connection.

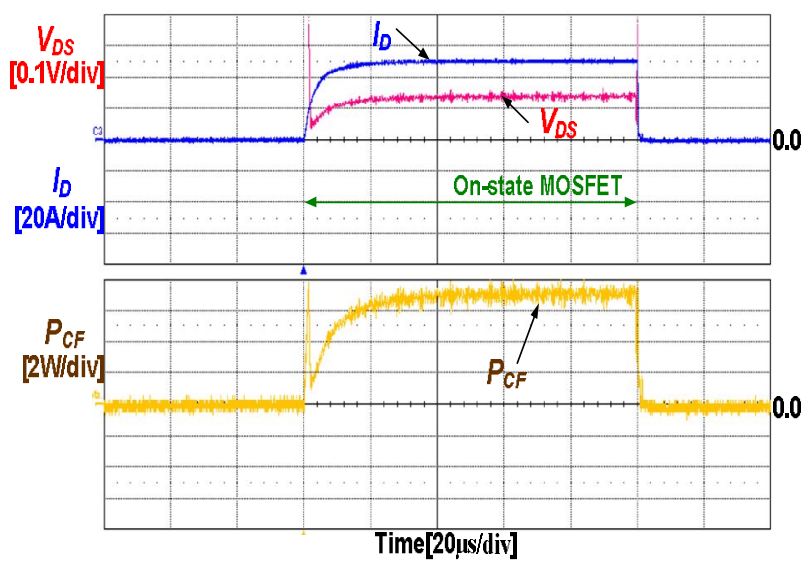

Fig. 4. Experimental result of conduction loss of MOSFETs at $I_{a}=50 \mathrm{~A}, n=5$.

Fig. 3 shows the plots of the conduction loss of all MOSFETs with a variation of $n$ under various armature currents at $r_{d s(o n)}=16.5 \mathrm{~m} \Omega$. As the $n$ is increased, the conduction loss of MOSFETs is significantly reduced. By considering both the conduction losses from Fig. 3 and the cost, the number of MOSFETs in parallel connection is determined on five. Thus, the conduction loss of MOSFETs can be reduced to about $30 \mathrm{~W}$ when the armature current is 100A. Fig. 4 shows experiment results of conduction loss of 
the MOSFETs obtained by multiplying $V_{D S}$ by $I_{D}$ at $n=5, I_{a}$ $=50 \mathrm{~A}$, where the conduction loss can be decreased to about $7.5 \mathrm{~W}$.

Assuming that the $m$ diodes are connected in the parallel, the armature current is shared with $m$ diodes. A forward voltage drop of the diode is slightly decreased as decreasing a forward diode current. Because the current of each diode becomes $\left(I_{a} / m\right)$, the forward voltage of a diode can be decreased. The total conduction loss of all diodes $P_{C D}$ can be calculated from multiplying the forward voltage dependent on one diode current by the armature current.

$$
P_{C D}=V_{F}\left(I_{a} / m\right) \cdot I_{a}
$$

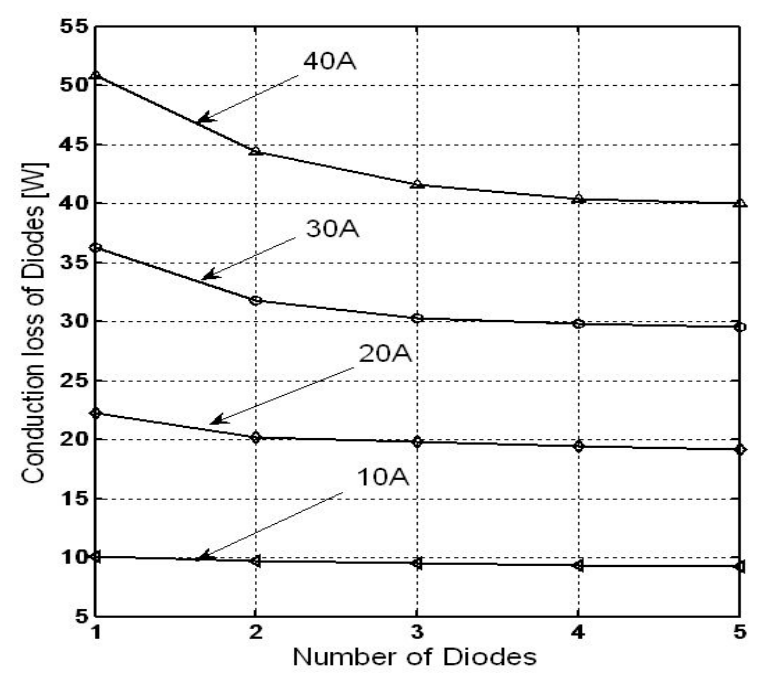

Fig. 5. Conduction loss with a variation of number of diode in parallel.

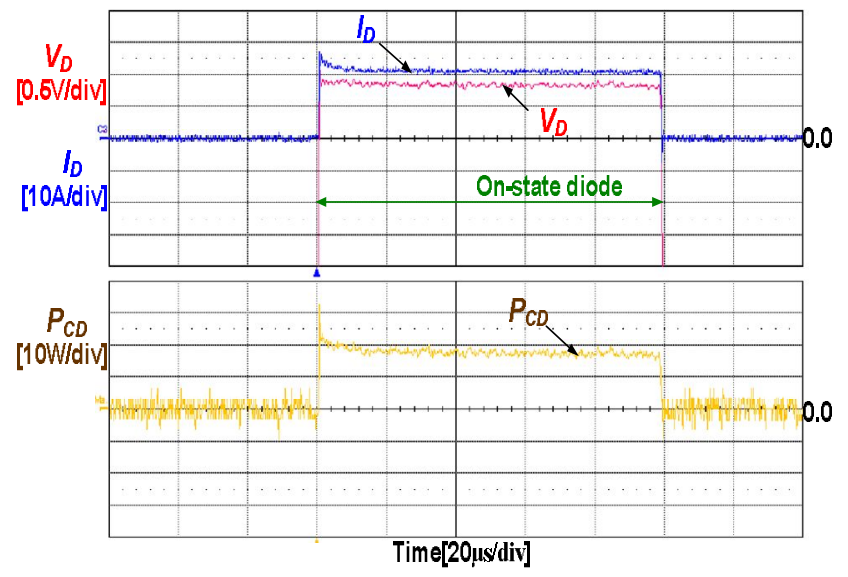

Fig. 6. Experimental result of conduction loss of diodes at $I_{a}=20 \mathrm{~A}, m=4$.

Fig. 5 shows the plots of the conduction losses of all diodes of the buck converter with a variation of $m$ under various armature currents. As the $m$ is increased to five, the conduction loss can be reduced to $10 \% \sim 20 \%$. In this paper, $m$ is determined as four, and the conduction loss of diodes can be reduced to about $3 \mathrm{~W}$ when the diode current is $20 \mathrm{~A}$. Fig. 6 shows experiment results of conduction loss of the diodes obtained by multiplying $V_{D}$ by $I_{D}$ at $I_{a}=20 \mathrm{~A}$.

The total conduction loss of the buck converter which consists of five MOSFETs and four diodes, is expressed as

$$
P_{C}=I_{a}^{2} \cdot\left(\frac{r_{d s(o n)}}{5}\right) \cdot d+V_{F}\left(\frac{I_{a}}{4}\right) \cdot I_{a} \cdot(1-d)
$$

,where $d$ is a duty cycle of the buck converter. Fig 7 shows plots of the conduction losses at $I_{a}=50 \mathrm{~A}$ when the duty cycle $d$ is variation form 0.5 to 1 . Because the conduction loss of the diodes is greater than that of the MOSFETs, the total conduction loss is $30 \mathrm{~W}$ at $d=0.5$. As a duty cycle increases, the total conduction loss is reduced to $8 \mathrm{~W}$.

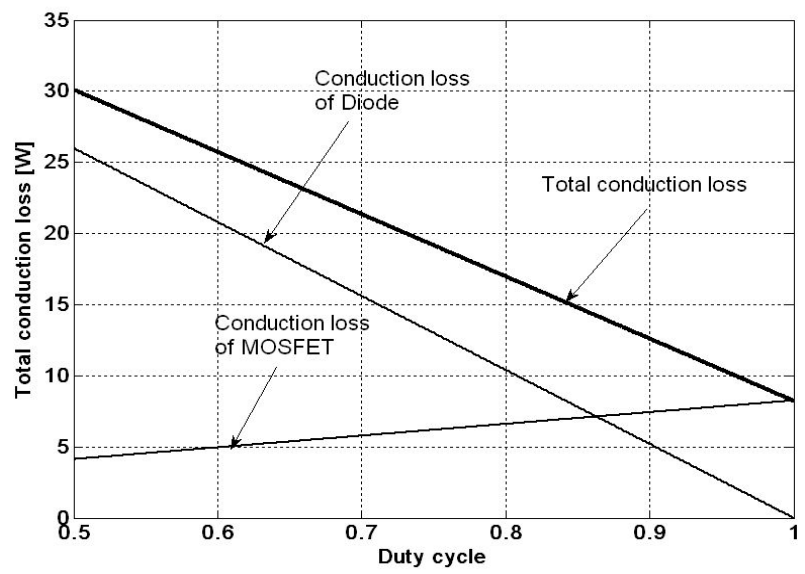

Fig. 7. Conduction losses with a variation of duty cycle at $I_{a}=50 \mathrm{~A}$.

\subsection{Switching loss of MOSFET}

The switching loss of all MOSFETs of the buck converter can be derived as

$$
P_{S F}=\frac{1}{2} \cdot V_{D S} \cdot I_{D} \cdot\left(t_{o n}+t_{o f f}\right) \cdot f_{S}+n \cdot C_{D S S} \cdot V_{D S}^{2} \cdot f_{S}
$$

where $t_{\text {off }}$ is the turn-off time, $t_{\text {on }}$ is the turn-on time, $f_{s}$ is switching frequency, $C_{D S S}$ is drain-source capacitance of the MOSFET.

Fig. 8 shows the waveform of $V_{D S}, I_{D}$ and switching loss at the turn-off and turn-on states at $V_{D S}=48 \mathrm{~V}, I_{a}=50 \mathrm{~A}$, $t_{\text {off }}=$ $800[\mathrm{~ns}], t_{o n}=120 \mathrm{~ns}, f_{s}=5 \mathrm{kHz}$, respectively. The switching loss is much lower than the conduction loss. 


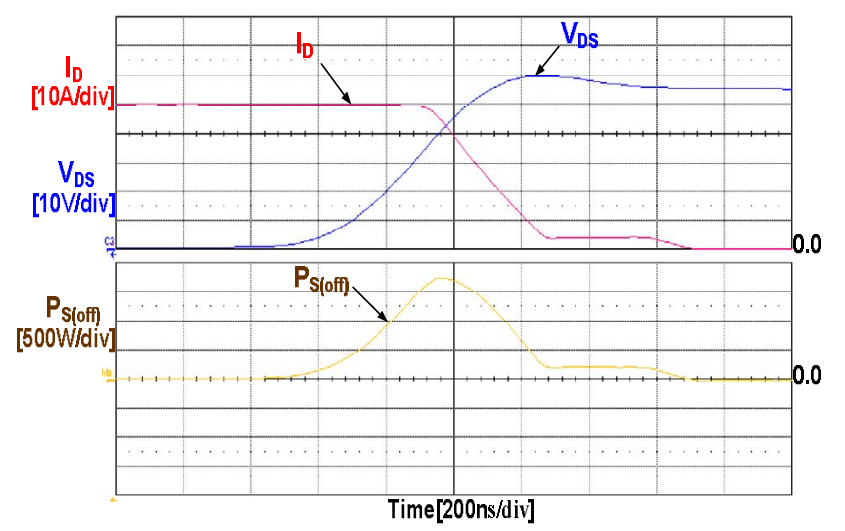

(a)

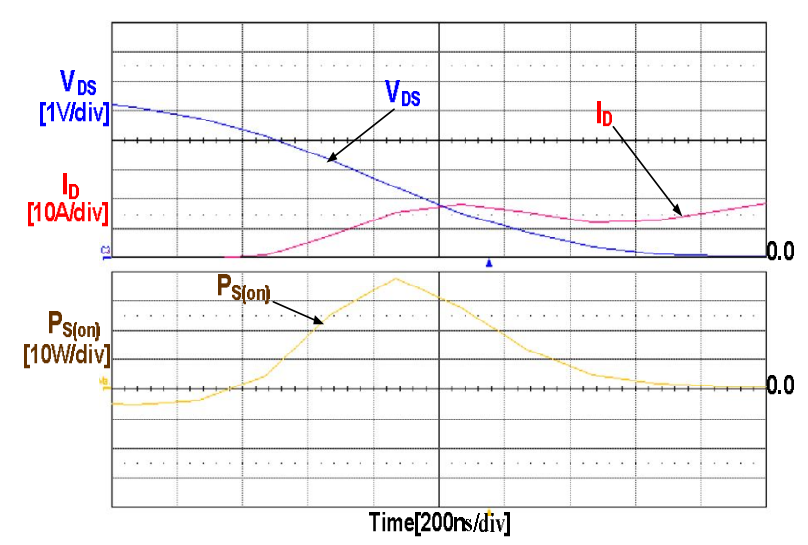

(b)

Fig. 8. Switching loss of MOSFET : (a) at turn-off state, (b) at turn-on state.

\section{Control scheme of DC Motor}

\subsection{Control algorithm}

The H-bridge type circuit is used to control the field current and also change the flowing direction of the current in order to reduce the cost of the power circuit. A rotating direction can be changed by changing a flowing direction of the field current. Before changing the rotating direction of motor, the field current as well as the motor speed should be gradually decreased to zero. However, as a motor speed is inversely proportional to the field current (flux), the motor speed increases rapidly to infinite when the field current approaches to $0 \mathrm{~A}$ while the armature voltage remains constant.

In order to change smoothly the rotating direction of $\mathrm{dc}$ motor, an operating sequence for both the field current and the armature voltage according to the accelerator pedal angle is proposed. Fig. 9 shows the operating pattern of the field current and motor speed according to the angle of accelerator pedal. Until the angle of an accelerator pedal reaches $10 \%$ relative to the maximum angle, the field current is kept to $10 \%$ relative to the rate current while the dc motor speed remains zero. When the angle approaches to $30 \%$, the field current goes rapidly to its rated value for generating the rated flux. The motor speed increases slowly, and goes to its rated speed at $100 \%$ pedal angle.

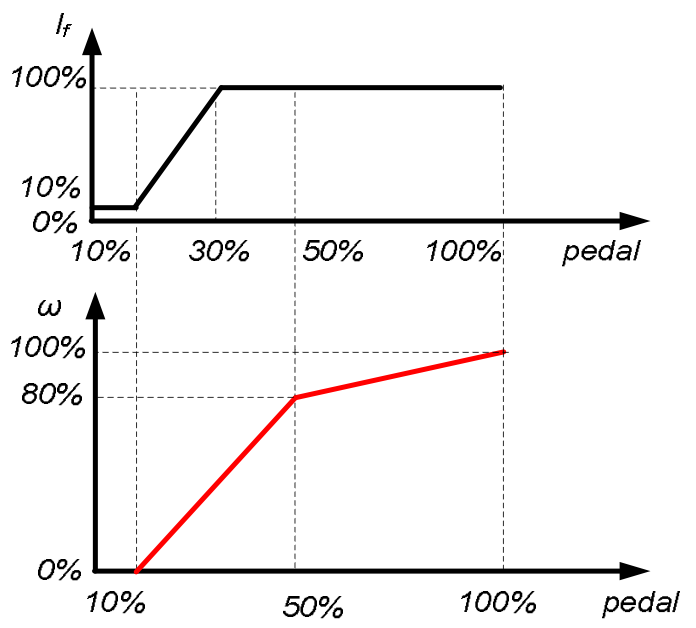

Fig. 9. Operating pattern for the field current and motor speed according to the pedal angle.

\subsection{Simulation result}

The simulation results are carried out to verify the performance for two different operating patterns when the rotating direction of motor is reversed. Fig. 10(a) shows the transient responses of the field current, armature voltage and current, the desired speed, and motor speed, when the field current goes to its rated value at $30 \%$ pedal angle and then the motor speed approaches to $80 \%$ relative to a rated speed at $50 \%$ pedal angle. Fig. 10(b) shows the same responses, when the field current goes to its rated value and the motor speed reaches to $80 \%$ at $60 \%$ pedal angle. It can be seen that the rotating direction of motor is smoothly changed, and the motor speed $\omega_{r}$ is nearly the same as the desired speed $\omega_{r}{ }^{*}$.

\section{Conclusion}

A high-efficiency and low-cost drive system of the smallsize EVs driven by the brushed dc motor was designed. The H-bridge type converter for field current control and the simple buck converter for armature current control were used for reducing the cost of drive circuit. The conduction loss of the buck converter for controlling the armature voltage can be reduced by less than $50 \%$ through the parallel connection of five MOSFETs and four diodes. The rotating direction of the dc motor was smoothly changed, when the proposed operating sequence for both the field current and the armature voltage was applied. 


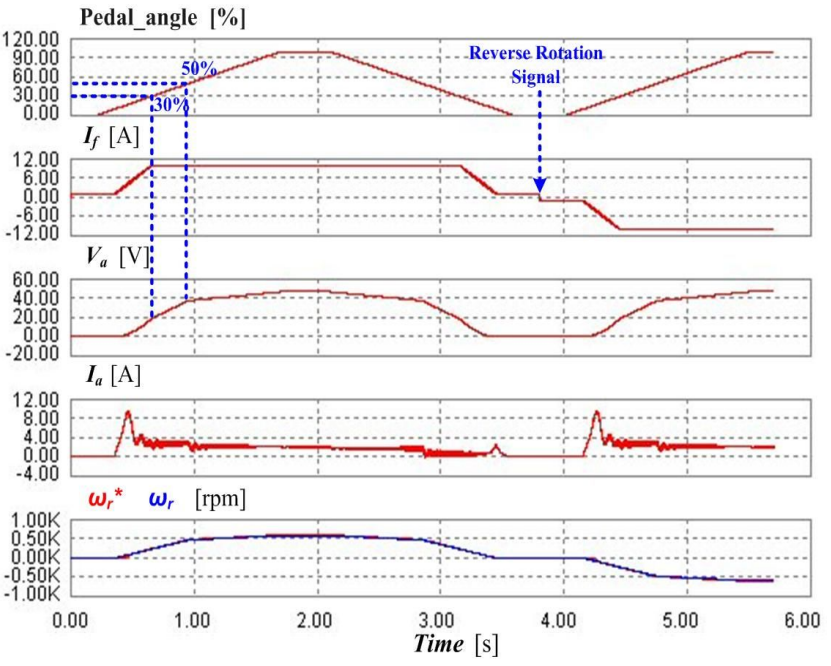

(a)

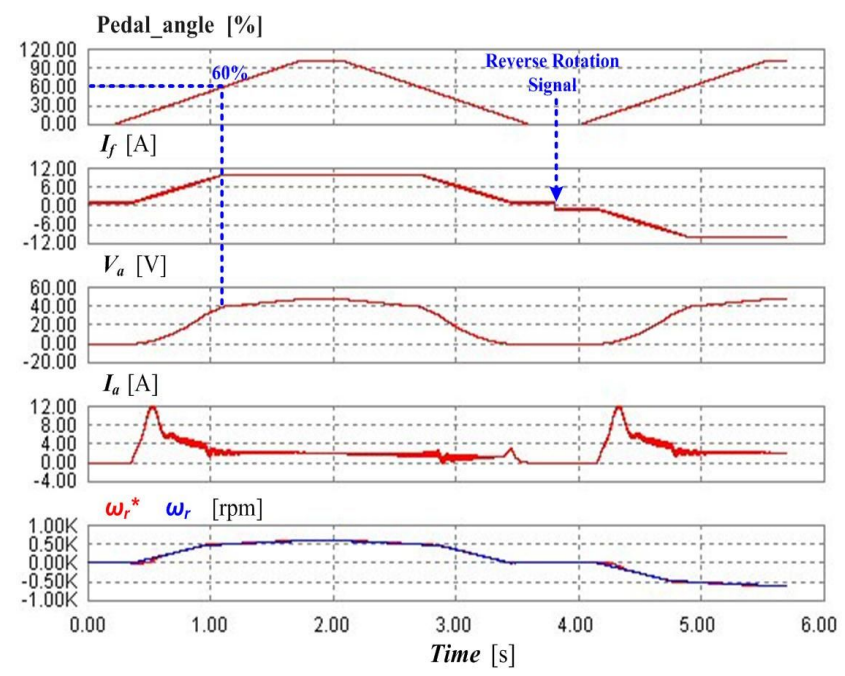

(b)

Fig. 10. Simulation results for two operating patterns at the rotation direction change.

\section{Acknowledgements}

This work was supported by School of Excellence in Electrical Engineering, University of Ulsan.

\section{References}

[1] M. Zeraoulia, M. EI H. Benbouzid, and D. Diallo, "Electric Motor Drive Selection Issues for HEV Propulsion Systems: A Comparative Study", IEEE Trans. on Vehicular Technologies, vol.55, no.6, pp. 1756-1764, Nov. 2006.

[2] D.G. Dorrell, A. M. Knight. L. Evans, and M. Popescu, "Analysis and Design Twchniques Applied to Hybrid Vehicle Drive Machines-Assessment of Alternative IPM and Induction Motor Topologies", in Proc. of IEEE-ISIE, 2011, pp. $1-10$.

[3] N.P. Shah, A.D. Hirzew, and B.H. Cho, "Transmissionless Selectively Aligned Surface-Permanent-Magnet BLDC
Moroe in Hybrid Electric Vehicles", IEEE Trans. on Industrial Electronics, vol.57, no.2, pp.669-672, Feb. 2010.

[4] Bharatkar, S.S., Yanamshetti, R., Chatterjee, D., and Ganguli, A.K., "Performance comparison of PWM inverter fed IM drive \& BLDC drive for vehicular applications", in Proc. of Vehicular Electronics and Safety, 2009, pp.125-129.

[5] M.S.Jayantha, C.F.Hayhoe, and J.J.Henry, "SCR-Controlled DC-Motor Model for an Electric Vehicle Propulsion System Simulation", IEEE Trans. on Industrial Electronics, vol.31, no.1, pp.18-25, Feb. 1984.

[6] Muhammad H.Rashid, Power electronic, circuits, devices and applications. 3'rd edition, Prentice Hall, 2004.

[7] Z.J.Shen, Y.Xiong, X Cheng, Y.Fu, and P.Kumar, "Power MOSFET switching loss Analysis: A new Insight”, in Proc. of IEEE-IAS 2006, pp.1438-1442.

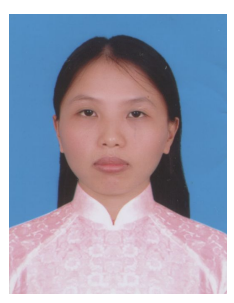

Thuy-Lien Duong was born in Vietnam in 1983. She received her B.S. degree in electronics and electrical engineering from Hochiminh University of Technology in 2006. From 2006 to 2009, she was with Hochiminh city Power Company at Vietnam. She received the M.S. degree in electrical engineering from University of Ulsan in 2012.

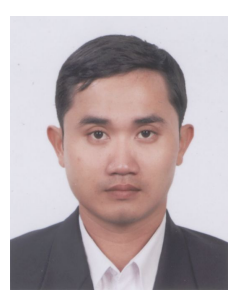

Thanh-Vu Tran was born in Vietnam in 1980. He received the B.S. degree in electronics and electrical engineering from Hochiminh University of Technology in 2005. From 2005 to 2009, he was with Hochiminh city Universtiy of Technology at Vietnam. He received the M.S. degree in electrical engineering from Hochiminh city Universtiy of Technology in 2008. He is currently working towards the Ph.D. degree in the electrical engineering, University of Ulsan.

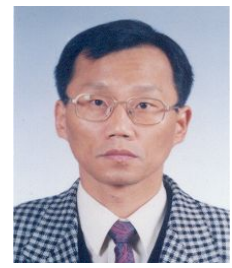

Tae-Won Chun was born in Korea in 1959. He received his B.S. degree in Electrical Engineering from Pusan National University, Korea in 1981, and received M.S. and Ph.D. degrees in Electrical Engineering from Seoul National University, Korea in 1983 and 1987, respectively. Since 1986, he has been a member of the faculty of the Department of Electrical Engineering, University of Ulsan, Korea, where he is currently a full Professor. From 1996 to 1997, he was with the Department of Electrical and Computer Engineering, University of Tennessee, USA as a visiting scholar. From 2005 to 2006, he also served as a visiting scholar with the Department of Electrical and 
Computer Engineering, Virginia Polytechnic Institute and State University, USA. His current research interests are the control of electrical machines and power conditioning systems.

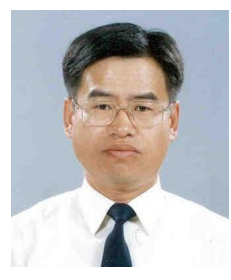

Hong-Hee Lee was born in Korea in 1957. He received the B.S., M.S. and Ph.D. degrees in Electrical Engineering from Seoul National University, Korea, in 1980, 1982 and 1990, respectively. Since 1985, he has been a member of the faculty of the Department of Electrical Engineering, University of Ulsan, Korea, where he is currently a full Professor. He was with the Department of Electrical Engineering, University of Texas A\&M, USA as a visiting scholar from 1990 to 1991. His current research interests are the wind power generation, matrix converters, and network-based factory automations.

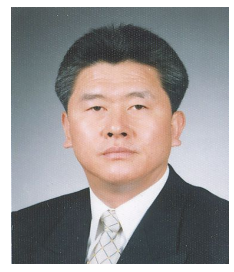

Heung-Gun Kim was born in Korea in 1956. He received his B.S., M.S. and $\mathrm{Ph} . \mathrm{D}$. degrees in Electrical Engineering from Seoul National University, Korea, in 1980, 1982 and 1988, respectively. Since 1984, he has been a member of the faculty in the Department of Electrical Engineering, Kyungpook National University, Korea, where he is currently a full Professor. He was with the Department of Electrical Engineering, University of Wisconsin-Madison, USA, as a visiting scholar from 1990 to 1991. From 2006 to 2007, he also served as a visiting scholar with the Department of Electrical and Computer Engineering at Michigan State University, USA. His current research interests are in the control of ac machines and PV power generation. 